\title{
Challenges and opportunities for telehealth in the management of chronic obstructive pulmonary disease: a qualitative case study in Greece
}

Violeta Gaveikaite ${ }^{1,2^{*}}$ (D), Casandra Grundstrom ${ }^{3}$, Stefan Winter ${ }^{4}$, Helen Schonenberg ${ }^{2}$, Minna Isomursu ${ }^{3}$, Ioanna Chouvarda ${ }^{1}$ and Nicos Maglaveras ${ }^{1,5}$

\begin{abstract}
Background: Telehealth $(\mathrm{TH})$ was introduced as a promising tool to support integrated care for the management of chronic obstructive pulmonary disease (COPD). It aims at improving self-management and providing remote support for continuous disease management. However, it is often not clear how TH-supported services fit into existing pathways for COPD management. The objective of this study is to uncover where TH can successfully contribute to providing care for COPD patients exemplified in a Greek care pathway. The secondary objective is to identify what conditions need to be considered for successful implementation of TH services.

Methods: Building on a single case study, we used a two-phase approach to identify areas in a Greek COPD care pathway where care services that are recommended in clinical guidelines are currently not implemented (challenges) and areas that are not explicitly recommended in the guidelines but that would benefit from TH services (opportunities). In phase I, we used the care delivery value chain framework to identify the divergence between the clinical guidelines and the actual practice captured by a survey with COPD healthcare professionals. In phase II, we conducted in-depth interviews with the same healthcare professionals based on the discovered divergences. The responses were analyzed with respect to identified opportunities for $\mathrm{TH}$ and care pathway challenges.
\end{abstract}

Results: Our results reveal insights in two areas. First, several areas with challenges were identified: patient education, self-management, medication adherence, physical activity, and comorbidity management. TH opportunities were perceived as offering better bi-directional communication and a tool for reassuring patients. Second, considering the identified challenges and opportunities together with other case context details a set of conditions was extracted that should be fulfilled to implement TH successfully.

(Continued on next page)

\footnotetext{
* Correspondence: gaveikaite@gmail.com

'Laboratory of Computer Science, Medical Informatics and Biomedical Imaging Technologies, School of Medicine, Faculty of Health Sciences, Aristotle University of Thessaloniki, 54124 Thessaloniki, Greece

${ }^{2}$ Department of Collaborative Care Solutions, Philips Research, High Tech Campus 34, 5656AE Eindhoven, The Netherlands

Full list of author information is available at the end of the article
}

(C) The Author(s). 2020 Open Access This article is licensed under a Creative Commons Attribution 4.0 International License, which permits use, sharing, adaptation, distribution and reproduction in any medium or format, as long as you give appropriate credit to the original author(s) and the source, provide a link to the Creative Commons licence, and indicate if changes were made. The images or other third party material in this article are included in the article's Creative Commons licence, unless indicated otherwise in a credit line to the material. If material is not included in the article's Creative Commons licence and your intended use is not permitted by statutory regulation or exceeds the permitted use, you will need to obtain permission directly from the copyright holder. To view a copy of this licence, visit http://creativecommons.org/licenses/by/4.0/. The Creative Commons Public Domain Dedication waiver (http://creativecommons.org/publicdomain/zero/1.0/) applies to the data made available in this article, unless otherwise stated in a credit line to the data. 


\begin{abstract}
(Continued from previous page)
Conclusions: The results of this case study provide detailed insights into a care pathway for COPD in Greece. Addressing the identified challenges and opportunities in this pathway is crucial for adopting and implementing service innovations. Therefore, this study contributes to a better understanding of requirements for the successful implementation of integrated TH services in the field of COPD management. Consequently, it may encourage healthcare professionals to implement TH-supported services as part of routine COPD management.
\end{abstract}

Keywords: Telehealth, COPD, Chronic obstructive pulmonary disease, Integrated care, Case study, Opportunities, Challenges

\section{Background}

COPD is a chronic disease associated with an increased incidence of morbidity and mortality worldwide [1]. COPD-related exacerbations, which often result in hospital re-admissions [2], have a substantial impact on the quality of life of patients as well as on healthcare resources [3]. To illustrate this, the COPD-related hospital admission rate in 2009 in the European Union was 184 per 100,000 persons a year, exceeding asthma-related admissions almost threefold [4]. To minimize the societal burden and improve patient outcomes, more effective disease management is necessary.

The World Health Organization describes effective COPD management as a prevention of disease progression, symptoms relief, improved exercise tolerance and enhanced health status, prevention and treatment of complications and exacerbations, and reduced mortality [5]. In other words, the goals of chronic disease management are not related to a cure, but rather to minimizing symptoms through secondary prevention and to enhancing both functional status and quality of life [6]. Traditional healthcare approaches that focus on a specific disease are failing to meet the requirements of effective management [7] because COPD patients are often multimorbid [8]. In this light, embracing a more patient-centered approach that includes patients and healthcare professionals (HCPs) working together to optimize disease management is favorable [7]. Therefore, improvements in healthcare processes and advances in technology, such as those that allow for the implementation of telehealth $(\mathrm{TH})$, are necessary developments [9].

$\mathrm{TH}$ is the ongoing and remote exchange of data between patients at home and healthcare professionals as part of disease management [10]. TH is expected to enable patient-centered care and offer solutions to tackle the problems of chronic disease management [7]. While early studies show that $\mathrm{TH}$ services reduce the utilization of healthcare services and the workload of HCPs, this is not a universally accepted truth [11-13]. In addition, $\mathrm{TH}$ is expected to promote and enable patient self-management [14]. Patient self-management is vital in chronic diseases management [15], but very challenging to implement in actual clinical care because of the organizational issues or education skill deficit [16].
Therefore, a strategy on how to promote and support patient self-management in actual clinical care should be developed and subsequently advocated.

One reason for the debatable impact of $\mathrm{TH}$ is the episodic care structure in healthcare systems. When considering care more continuously, integrated care (IC) and value-based care (VBC) are paradigms that try to tackle this problem through process innovations [17]. IC focuses on process innovations and value-case development [18]. This allows the advancement of a shared vision and setting common goals across different providers or teams. A value case looks beyond the potential financial returns of an individual stakeholder towards the benefits for patients and the community as a whole [18]. In VBC theory, value is defined as the health outcomes that matter to a patient over a complete cycle of care, per dollar spent [19]. A clear advantage of VBC is availability of strategic toolset for implementation in actual clinical care [17]. For example, adequately named care delivery value chain (CDVC) offers a systematic framework to delineate and analyze the process of care delivery for a specific medical condition. This framework helps to understand the diverse aspects of care delivery, how disease relates to other care processes, and where structural improvements can be made [19].

One strategy to improve care delivery is the creation or improvement of a care pathway. Care pathways are structured multidisciplinary plans which coordinate essential steps in the care of patients with a specific clinical problem [20]. There have been several attempts in Europe to build COPD care pathways. However, they currently do not incorporate TH services at scale [21]. This may be explained by the limited evidence for positive outcomes related to $\mathrm{TH}$ services implementation of $\mathrm{TH}$ services into the actual clinical practice presented in current clinical practice guidelines [2].

A recent COPD care pathway mapping effort showed five European macro care pathways [22]. Interestingly, the five care pathways were quite different when considering them from the perspective of patient referral systems, the maturity of technological infrastructure, and stakeholder involvement [22]. Country-specific challenges require more innovative approaches, such as $\mathrm{TH}$, 


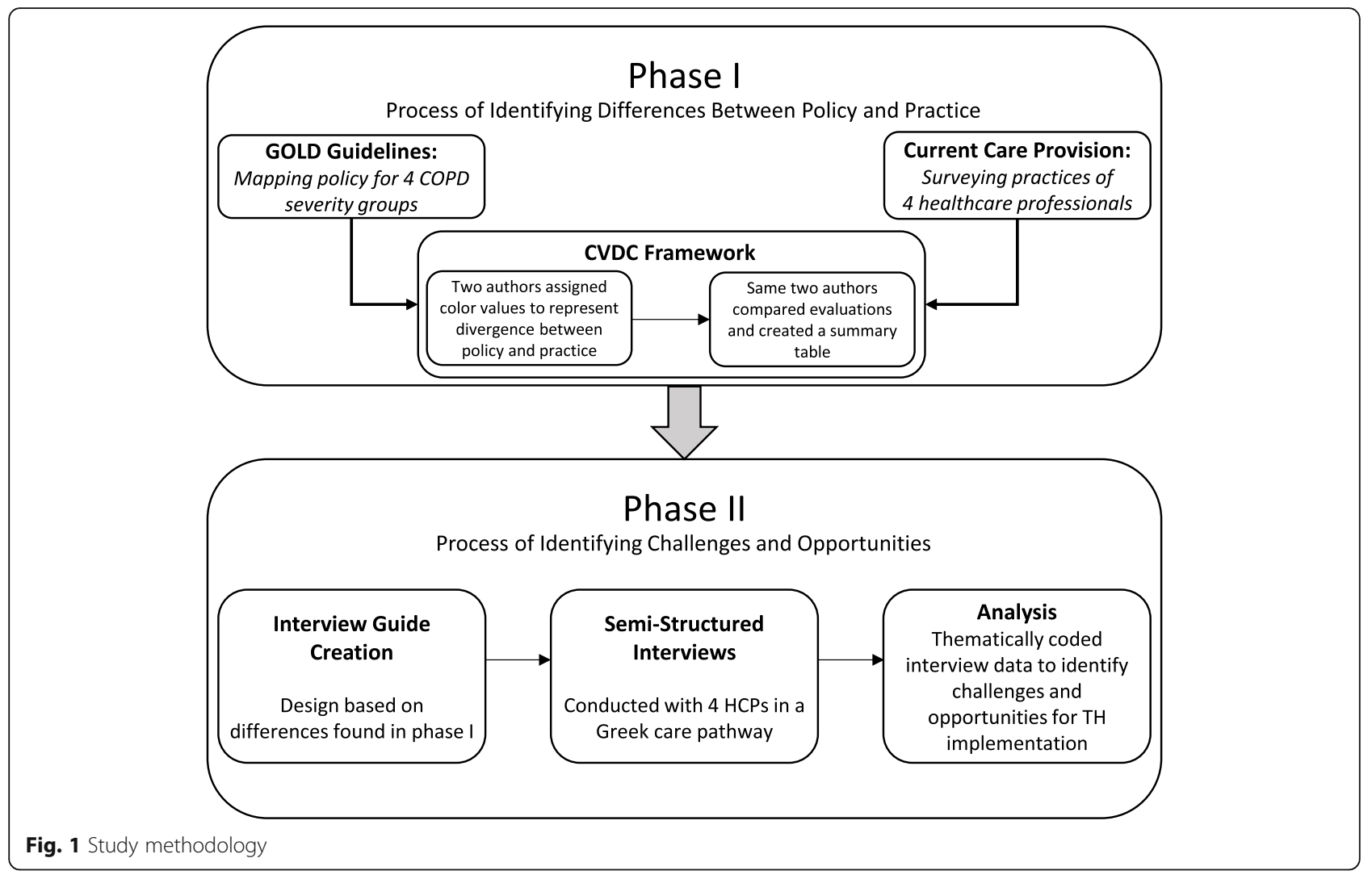

to improve IC and enable patient self-management. This study will contribute to the research stream by investigating one such care pathway in more detail with a focus on pinpointing the potential advantages of $\mathrm{TH}$ services. This paper addresses the following research question: What opportunities for $\mathrm{TH}$ and challenges are perceived by HCPs in a COPD care pathway?

To address this research question, we use Greece as a country-specific context to focus on a specific COPD care pathway as a case study. We explore the perspectives of four HCPs by combining different qualitative data collection methods to gain better understanding of what conditions need to be facilitated to successfully implement $\mathrm{TH}$.

\section{Methods}

\section{Definitions}

A challenge is defined as a care service that is recommended in clinical guidelines but missing in current clinical practice. An opportunity is defined as a TH care service that is not present in current clinical guidelines or practice but is expected to bring benefits or value to relevant stakeholders. An innovation is defined as a TH care service that either contributes to solving a challenge or introduces an opportunity.

\section{Case study}

The study methodology is depicted in Fig. 1. For the purposes of this study, the instrumental case study approach was selected as it is appropriate to generate an in-depth appreciation for a particular situation [23]. The flexibility of a case study approach is uniquely suited for the goals of this project, as it offers a natural understanding of the complex phenomena of practices and perceptions in a care pathway [24]. This case study was performed in the Asclepius hospital (Thessaloniki, Greece) $)^{1}$, which is a referral center for respiratory diseases in Greece. Four different HCPs have been surveyed and interviewed (Table 1). More detailed participant characteristics including job description and tasks have been published previously [25]. The governance structure of the Asclepios hospital could be defined as hierarchical, where top-down leadership is present in all levels of the organization. The director is appointed by the health minister and supported by a management board that includes medical doctors. The diagnosisrelated group system is not yet present. However, it is under active central development and will be tested soon. The budget is fixed and defined by the management

\footnotetext{
${ }^{1}$ For ease of referencing, we will refer to the case study site as 'Asclepius' for anonymization purposes, and to prevent revealing the identity of the hospital and/or staff.
} 
Table 1 Participant characteristics in Greece care pathway

\begin{tabular}{lll}
\hline Participant ID & Job Title & Job Location \\
\hline 1 & Head Nurse & Public healthcare hospital \\
2 & Physiotherapist & Public healthcare hospital \\
3 & Pulmonologist (in training) & Public healthcare hospital \\
4 & Pulmonologist & $\begin{array}{l}\text { Private practice with referrals } \\
\text { to public healthcare hospital; } \\
\end{array}$ \\
& the gate keeper \\
\hline
\end{tabular}

Participant ID number corresponds to answers in the results section

board. There are frequent budget cuts and poor flexibility due to the ongoing economic crisis in Greece. In lower management levels, self-governance is present at the department level. Quality controls are implemented through a top-down approach and governed by doctors. There is a lack of performance-based evaluation, which means that there are no annual goals or targets set at any level in the organization.

The hospital is organized in three parts for treating respiratory conditions, including COPD: in-hospital unit, emergency department (ED) and outpatient clinic. It was not possible to retrieve the number of COPD-related visits to the hospital. There were about 5000 respiratoryrelated outpatient clinic visits in 2016, approximately 2400 ED admissions and 2100 patients were hospitalized. Mean hospital admission duration was 4.2 days. The inhospital respiratory department consists of 6 doctors, 12 permanent nurses, several nursing staff assistants, one physiotherapist and one spirometry specialist. The department is an active regional participant in scientific research and actively involved in providing training programs for a variety of clinical trainees/residents.

The participant selection process was based on the COPD care pathway reported in relevant literature in Greece; each participant represents one of four major HCPs in the care pathway [22]. In Greece, the pulmonologists have a "gatekeeper role" for stable COPD patient management which means, that in case of any problems patients first consult the pulmonologist, who in turn can refer them to other specialists such as pulmonary rehabilitation specialists. Patients with exacerbations are treated in the hospital, followed by inpatient or outpatient care during follow-up visits [22].

The four HCPs are the primary stakeholders involved in the care of patients with COPD in Greece. They are often participating in research projects which are designed to deliver remote care [22]. Professional help with social or psychological issues is not a common practice in Greece, as family members traditionally play a significant role in patient care. However, psychiatric care is part of comorbidity management in this care pathway. In the Asclepius hospital, the average COPD caseload is around 15-20 patients per week. The hospital has a catchment area that includes the urban areas of Thessaloniki as well as more remote, rural areas. The public healthcare system is the focus of the case study. Convenience sampling was used to recruit HCPs [26]. All healthcare professionals are actively participating in the care pathway for COPD patients and permanently employed at the Asclepius hospital.

\section{Data collection and analysis}

Data collection and analysis this case study was a sequential process of two iterative phases with an increasing level of detail, where data was both collected and analyzed in each phase.

\section{Phase I - identification of divergences between policy and practice to inform the creation of an interview guide} The goal of this phase was to prepare for the creation of the interview guide. First, the GOLD guidelines were mapped into the CDVC framework (policy CDVC). Second, HCPs filled in the CDVC framework based on their actual practice (practice CDVC). Third, the divergence between policy CDVC and practice CDVC was used to inform the interview guide creation process.

National COPD guidelines are used to evaluate the policy context for this case study which took place during March 2018. We adopted the CDVC framework as a mapping tool to understand the divergence between the clinical guidelines and existing evidence-based care practices [19]. The mapping of the guidelines allowed for a structured and systematic approach to understanding the different care processes for each COPD severity. The use of surveys is a common tool accepted by HCPs as a flexible and non-intrusive method of gathering data [27]. The survey retained the original CDVC structure which corresponds to six areas of our interest. These areas are as follows: monitoring and prevention, diagnosis, intervention preparation, intervention, recovery and rehabilitation, and monitoring and management. The survey asked which clinical guidelines were followed by the participants, if any, as well as provided examples for COPD care considerations for informative purposes. The survey was color-coded to allow for ease of reading and structured filling out. The survey template is available in Additional file 1. Surveys were distributed to the HCPs during March 2018. The completed surveys were collected in June 2018. Four HCPs from along the COPD care pathway in Asclepius hospital (see Table 1) filled them out individually. To further allow for triangulation - the mixing of data types from multiple sources to facilitate validation [28] - all additional documentation provided by the clinical participants were included in the analysis process. The divergence between policy and practice was determined by matching the guidelines (Additional file 2) to the practices of the healthcare professionals. To perform this task, two authors (VG and 
CG) individually assigned color values to represent the completeness of the practices against policy for each HCP. Then the same two authors compared their individual evaluations and created a summary table, which depicted the level of which healthcare provision was compliant with policy (Additional file 3). To depict compliance, stop-light color coding was used. Observed in Phase I were used to focus the semi-structured interviews in Phase II.

\section{Phase II - interview guide creation for challenges and opportunities}

The goal of this phase was to explore the challenges and opportunities for the implementation of $\mathrm{TH}$-supported services in the Greek care pathway. First, a semistructured interview guide was designed. Second, interviews were conducted with the same four healthcare professionals who were surveyed in Phase I. Third, interviews were thematically analyzed for challenges and opportunities of $\mathrm{TH}$ implementation.

The semi-structured interview guide was designed based on the divergence between policy and practice in Phase I. The four areas which showed a significant discrepancy between policy and practice were: diagnosis, intervention preparation, intervention, and recovery and rehabilitation. The semi-structured interview guide is available in Additional file 4. No specific TH interventions were mentioned during the interview to prevent influencing the HCPs' perception of TH.

The semi-structured interviews were conducted at pre-arranged and mutually convenient times and lasted between 30 and $60 \mathrm{~min}$. The interviews closely followed the interview guide to ensure homogenous data collection. All interviews were transcribed following a denaturalization process to remove language idiosyncrasies while also remaining faithful to meanings and perceptions of the interviewee [29]. The framework method from Gale et al. [30] was followed to ensure an organized and systematic coding process, leading to the generation of meaningful themes. The theming process and analysis was performed without specialized software. The framework method was chosen as it is an appropriate management tool for multi-disciplinary research teams in health service research, and provides structure during the analysis of qualitative data [30]; important for our study due the varying levels of qualitative experience.

\section{Results}

The study type is considered to be instrumental, meaning Phase I results are used towards informing the main results section in Phase II.
Phase I - groundwork for the interview guide creation In Asclepius, the main COPD care document is the GOLD guidelines [31], which is used by all HCPs. Patients are classified based on the impact of COPD on the patient using the recently introduced $\mathrm{ABCD}$ assessment tool. This tool combines the assessment of symptoms with the patient's spirometry classification and/or risk of exacerbation [31]. The guidelines indicating COPD policy are available in Additional file 2.

The differences in clinical care processes between clinical practice and clinical guidelines are available in Additional file 3. The two areas proven to match well between policy and practice were: monitoring and prevention before COPD diagnosis, and monitoring and management after the patient is discharged. Three areas, namely diagnosis, intervention, and recovery and rehabilitation, only partially matched between policy and practice. The cross-section representing interventions for very severe patients, was found dissimilar to policy guidelines. Finally, clinical participants did not mention the preparation of interventions.

\section{Phase II - perceived challenges and opportunities Challenges within self-management and comorbidity management}

In this section, we present the challenges within five key areas: patient education, medication adherence, physical activity, smoking cessation, and comorbidity management. Self-management includes patient education, medication adherence, physical activity and smoking cessation.

Patient education In Greece, both the pulmonologist working in the hospital and the private practice specialist teach dyspnea management and coughing techniques during patient visits or distribute take- home materials. In Greece, a hot and humid country, weather-based events are significant environmental factors for HCPs to consider. Both medical doctors highlighted that they advised patients during visits on climate-related issues: "It's very difficult for them. During the summer, the temperature is very high ... we instruct them to go outside or to do their shopping early in the morning or late at evening. And here especially in Thessaloniki, we have a lot of humidity, which is something that might impact the respiratory system" (4 - Pulmonologist). Awareness about issues in winter are also stressed: "I give the instructions during the winter [regarding] the fireplaces..." (4 - Pulmonologist).

The interviews suggest that the role of clinicians in patient non-pharmacological management was to provide the necessary information and clear recommendations to patients. The pulmonologist expressed concerns about the efficacy of paper flyers and whether or not patients actively participate in self-education at home. "Most of 
[the patients] do not even read [the flyers]. We are asking and we are trying to see if they are doing everything we discussed in the previous visit" (4 - Pulmonologist). Considering both roles, a healthcare provider distributing take-home materials in the form of flyers indicates that only verbal and passive patient education takes place. The majority of patients do not show self-sustaining home-behaviors for self-education.

A problem discussed by the HCPs is the relative lack of doctors considering the number of patients. This means HCPss must prioritize topics to discuss with the patient, which may not always include support for selfeducation: "It's difficult, we have too many patients and we are very few doctors... We don't have ability to tell them many things" (3 - Pulmonologist). The challenge within education is found in conveying impactful education on a large scale that is meaningful for COPD patients.

Medication adherence Doctors are responsible for informing the patient on correct medication usage (informing self-management), including inhalation techniques. However, adherence to medication and proper use outside of the hospital is the responsibility of the patient (sustaining self-management). Our interviews revealed that the emphasis on medication adherence support is adopted by clinicians to 'motivate' patients. The practice of informing about self-management for medication adherence requires doctors to allocate specific time during the patient visit. A nebulizer is a drug delivery device that requires inhaler technique training provided during the patient visit. As the inhaler technique training is extensive and demands specific skills, the time it takes to inform about, train, and practice the correct technique is quite high. "When I start to give an inhalation device to my patients, first thing I do I explain how it works and I have a device for demonstration they can [practice on]" (4 - Pulmonologist).

However, ensuring that the tool is being used correctly at home, relies on the patient's understanding during the patient visit. "I ask to show me how they take the medication. If they don't take them properly, I have a device for demonstration to show them again and again how they can take medicines" (3 - Pulmonologist). This illustrates that medication adherence at home is at least partially dependent on doctors informing patients about it during the visit.

Administering the correct dosage of medication when the patient is at home is also important for COPD selfmanagement. The doctors rely upon patient recollection through feedback prompting to paint a picture of adherence. "I ask them how many times they take a medication a day..." (3 - Pulmonologist). Forcing the doctors to trust what the patient is reporting and relying on the accuracy of subjective evaluation. "You have to believe what they saying to you, but if the patient doesn't do exactly what are you [advising]" (4 - Pulmonologist). However, both doctors reported that they might be suspicious of poor medication adherence for a variety of reasons. For example, the correct usage of an inhalation device if the patient is showing symptom deterioration. To support medication adherence at home, no technology tool is available. The challenge within medication adherence is how to both inform on and sustain patient self-management.

Physical activity The physiotherapist by training, and pulmonary rehabilitation specialist by title has the primary responsibility for physical activity management of the care pathway in Asclepius hospital and provides an unstructured rehabilitation program. Asclepius adheres to the practice of directing patients to pulmonary rehabilitation specialists through a set process. Both medical doctors reported that a physiotherapist should provide information on physical activity, as care beyond the walls of the hospital is not part of the job scope: "Here in Greece we don't have [physical activity training] ... we refer them to physiotherapist" (4 - Pulmonologist).Physical activity programs do exist in some Greek hospitals, but the doctors interviewed do not know anything about it: "There is such a program in another hospital, but I don't participate, so I can't tell you about that" (3 Pulmonologist).

The physiotherapist expressed that although physical activity is a crucial part of managing stable COPD, other priorities take place in their work practices such as informing on cough, dyspnea, and sputum production. "Physical activity is very important but when [the] patient comes to the hospital the first [focus] is the cough, not a physical activity, this is after" (2 - Physiotherapist). Part of the reason why sustaining remote physical activity practices are not in place is because the physiotherapist cannot visit the home of the patient. Instead, the burden of choice for physical activity is placed squarely on the shoulders of the patient. "[The patient] can decide if he wants to go to[a] private clinic or to [a] place to continue [the physical activity]" (2 - Physiotherapist). The physiotherapist informs on using a static bike at home either in bed or in a chair when the patient is hospitalized.

Apart from not being able to visit the patient at home, the physiotherapist also cannot prioritize providing physical activity consultations due to more critical selfmanagement elements of which they are responsible "... the cough and the dyspnea [are] the most important" (2 - Physiotherapist). The challenge within physical activity is providing physical activity to COPD patients in either the rehabilitation or home setting. 
Smoking cessation The interviews revealed that in the public hospital no information on, or follow-up for smoking cessation is provided. In Greece, smoking cessation intervention is the responsibility of smoking cessation clinics and pulmonologists trained to provide smoking cessation services. In the care pathways of Asclepius, smoking cessation specialists are not part of care. "We don't have specialized doctors. And we advise them to visit [a] specialized doctor and this topic" (3 Pulmonologist).

Even though the pulmonologist is specialized in smoking cessation, these services are only performed in a separate, private clinic specializing in pulmonary rehabilitation. The pulmonologist mentioned and actively supports smoking cessation for COPD self-management, but the doctors have limitations. "If you want to manage smoking cessation, you need to be specialized as [a] pulmonologist. If you are not specialized pulmonologist in the smoking cessation you ... have to tell the patients [to] go [to the smoking clinics] for smoking cessation ..." (4 - Pulmonologist).

The nurse and physiotherapist have no role in supporting or making decisions on smoking cessation. Additionally, the pulmonologist receives limited feedback when a smoking intervention is carried out. Instead, all responsibility for smoking cessation shifts to the patient as no informing or sustaining activities are taking place during patient visits or hospitalization events. The challenge within smoking cessation is facilitating access to smoking cessation programs, and follow-up after the program takes place.

Comorbidity management The main three comorbidities that were found to significantly impact COPD management were: diabetes, heart failure, and depression. The nurse and the physiotherapist are not part of managing, treating, or making decisions related to comorbidities. "[We] wait for a doctor to give instructions, what to do with medications and the care with a patient" (1 - Head Nurse).

When dealing with comorbidities, the doctors were shown to be meticulous to check patient comorbidities "I ask them every time about their medical history, even if I know medical history, I ask them again, and again every follow up. I want them to show all medications they take, how many times a day" (3 - Pulmonologist). Some collaboration efforts are in place to ensure comprehensive patient treatment "we work together with a specialist. For example, if I have a COPD patient with heart failure, I would talk with cardiologist so to manage therapy protocol together for the heart failure and for COPD." (4 - Pulmonologist).

The participants indicated a lack of electronic records (EHR) means that most work is still done manually and in paper format. The challenge within comorbidity management is administration for tracking and managing a complex chronic disease with additional HCPs.

\section{Opportunities to support patients}

Telehealth as a bi-directional communication tool Participants mentioned that $\mathrm{TH}$ would facilitate more efficient access to patients: "It will be easier for the doctor to access the patient, and it would be easier for the patient to access the doctor" (4-Pulmonologist). The most important factor to highlight here is that access takes place in a bi-directional setting; the patient should have access to the doctor, and the doctor should have access to the patient. At the moment, the communication is initiated by the patient and usually triggered by a disease-related event.

The pulmonologists emphasized a potential shift towards patients taking more responsibility for their health because of $\mathrm{TH}$ intervention support and the capability to contact HCPs in case of a significant worsening of condition: "I want him to call me when he has very severe symptoms" (3-Pulmonologist). The TH system should also automatically notify HCPs when the clinical condition of a patient deteriorates: "Or the system to call me when these symptoms [change]..." (3-Pulmonologist).

The pulmonologists expressed a desire to stay in control of final decision making, meaning a TH system should support the capability of a decision maker but be limited to triage: "I don't want the system to decide what is more important for a patient" (4- Pulmonologist).

$\mathrm{TH}$ was also seen as a tool for HCPs to reduce the time intervals between appointments with patients: "[I] don't need to wait for an appointment with a patient to see if he has a problem. With telco, [I] can directly give advice [to patients]" (2- Physiotherapist). When considering remote areas where healthcare resources are scarce, having access to $\mathrm{TH}$ services would facilitate regular patient-doctor contact: "Especially here in Greece, we have areas that are very far away, and they don't have doctors and they don't have nurses and so the TH will be able to stabilize it" (1 - Head nurse). Healthcare service delivery and experiences for all stakeholders could be improved by $\mathrm{TH}$.

Telehealth as a tool to reassure patients Asclepius mainly manages severe cases of COPD, and many COPD patients have a sensitive psychological state, with depression being one of the most frequent comorbidities. The unexpected nature of exacerbations, more common in severe cases of COPD, keep patients in a constant state of anxiety: "Don't forget that these patients with stage three or four, they are very anxious about their health, if they call to the doctor, [the] doctor needs to immediately answer they call" (3 - Pulmonologist).Participants shared considerations that $\mathrm{TH}$ would help reduce patient anxiety because HCPs can monitor and respond to a patient's status: "So if you have telehealth system for these 
patients it would be better. Because they would know that the doctor has access to their data all the time. [The patients] know that the doctor will call them, or they can call the doctor to manage their problem" (3-Pulmonologist). It was further emphasized by all participants that TH interventions would increase patient-safety as well.

\section{Discussion}

\section{Challenges and opportunities in providing care for COPD} patients

There is limited qualitative research available, which details care pathway challenges and opportunities to empirically inform about possible care service solutions, such as TH. Our study contributes to this research area by illuminating the divergence between clinical guidelines and actual clinical care. There are four particular areas of clinical guidelines which should be emphasized for future TH implementation in the Greek care pathway. These are: diagnosis, intervention preparation, intervention, and recovery/rehabilitation. Self-management components for care provision are the most challenging and require a different organizational approach to be implemented and enforced in the actual care pathway. However, when comparing current literature with our findings, there is a striking difference as most studies focus on pharmacological management or medication adherence [32, 33]. Our research was focused on a nonpharmacological management for COPD. For instance, a study in Greece reported a low adherence to guidelines regarding treatment and highlighted several reasons for non-adherence [33]. It suggests that non-pharmacological management was not considered to be a care element.

Current practices may harbor prospects for TH implementation through the five challenges identified within self-management and comorbidity management for the COPD care provision. These were: education (conveying effective and impactful education on a large and meaningful scale), medication adherence (informing and sustaining patient medication behaviors), physical activity (facilitating rehabilitation in outpatient settings), smoking cessation (providing support for smoking cessation programs), and comorbidity management (managing administrative duties for a complex network of HCPs). All these elements are discussed in the GOLD guidelines [2]. It is important to emphasize, that these elements are discussed as individual elements. It is not yet clear how they can be combined. Some systematic reviews address that a self-management plan and its technical support might be beneficial for COPD patients. However, the evidence is not yet conclusive $[34,35]$ We suggest there is a need for research to determine not only the efficacy of TH for COPD management but also to utilize qualitative research to aid in the understanding of adoption and sustainability mechanisms when considering pathways and their features.
Difficulties in educating patients were seen as a communication challenge by clinical stakeholders due to a lack of effective and large-scale means to reach patients. This indicates a prospect for $\mathrm{TH}$ implementation because it allows remote patient education in large groups, does not require time scheduling and allows HCPs to sustain patient interest in self-management through education. A recent systematic review supports this finding, showing that education of chronic patients delivered through virtual modalities was comparable, or more effective than standard care [36]. This is important, since it demonstrates effectiveness of virtual education for chronic patients. The next step would be a large-scale implementation.

HCPs reported that informing and sustaining patient medication behaviors was a challenging task because the patient has a disease with complex medication needs. TH could support medication adherence because it offers a chance to track patients remotely. For instance, some trials incorporated the medication adherence report scale in their trial [37] or their daily symptom diary [38]. However, systematic knowledge towards managing medication adherence for COPD with $\mathrm{TH}$ tools is not present. This could be in large part due to few clinical trials using $\mathrm{TH}$ that report on medication adherence. Since there is a significant lack of evidence to support medication adherence with $\mathrm{TH}$, future trials investigating $\mathrm{TH}$ should include medication adherence as an outcome.

Facilitating physical activity for COPD is significant part of rehabilitation because it is a strong predictor of COPD patient mortality [39]. Our physical therapist considered $\mathrm{TH}$ to be useful in the outpatient setting as it improves their access to patients and increases patient activation. Considering physical activity support by $\mathrm{TH}$, Lundell et al. stated that TH may increase patients physical activity levels [40]. Moreover, recent studies show the potential of $\mathrm{TH}$ to increase or maintain physical activity when managing COPD patients [41, 42].

In COPD, smoking cessation is one of the most critical steps to positively influence the disease course [2]. In the Greek care pathway, the challenge within smoking cessation is restricted as HCPS have limited ability to provide smoking cessation programs. This finding is in line with report released in 2011, which stated that only 3\% smokers ever visited a smoking cessation office [43].

In addition, if the patient was referred to the smoking cessation clinic, his "treatment" outcome and follow-up was fragmented and did not reach the COPD care gatekeeper. TH would be a candidate to support such a service, as it provides remote access, follow-up possibilities and timely communication to boost patient motivation. In addition, sufficient clinical evidence is available to support the use of web -based smoking cessation 
programs for adult smokers in clinical practice [44]. Before implementing this solution in $\mathrm{TH}$ services, contextual factors should be considered.

Implementing web- or mobile application-based $\mathrm{TH}$ solution here could be an option, as it would provide timely access to the data for all stakeholders and facilitate decision making towards medication prescription or the COPD-exacerbation related diagnosis. However, there is little evidence to conclude that comorbidity monitoring is beneficial. Despite the lack of evidence, Bourbeau et al. propose to explore the area of the integrated remote monitoring of COPD-related comorbidities [12].

Bi-directional communication and $\mathrm{TH}$ as a tool to reassure patients were reported by our participants as opportunities for $\mathrm{TH}$ services. Our findings were consistent with opportunities reported in other studies. For instance, Ure et al. [45] discussed the importance of timely communication to prevent adverse disease outcomes, although bi-directional communication was not emphasized. The communication advantages were reported from different perspectives where patients felt happy with the chance to communicate with their HCP's. However, some nurses felt that TH limits their ability to understand the patient's condition and needs [46]. The nurse in our case study contradicts this attitude, and instead sees $\mathrm{TH}$ as a tool to facilitate better and different patient relationships [25]. Regarding our case, Greece has a lot of remote territories (up to 25\%) and TH could be used as a service to secure patientHCP communication, especially when considering medical staff shortages and economic crisis [47]. Huniche et al. [48] discussed "a sense of security" as a positive patient experience. However, it might be a misleading one, as it transfers responsibility away from HCPs, and towards the TH service.

\section{Conditions for implementing Telehealth}

Before implementing $\mathrm{TH}$ in routine care, multiple dimensions such as structure, process, and patient outcomes should be considered $[49,50]$. Structure describes the stable elements of care organizations (for example, facilities, education or technology). Within this dimension, four elements are worth mentioning in the Greek care pathway: the impact of the current economic crisis, IT infrastructure, the gatekeeper role for COPD care, and specialized nurse integration in the care organizational model.

The healthcare IT environment in Greece is limited for a variety of reasons. EHRs are not fully integrated into the system [51], which results in difficulties with care coordination and timely information transfer [52]. There is no policy to encourage the adoption and maintenance of high quality EHRs. The current economic crisis in Greece may shift healthcare priorities to other areas of interest [53]. In addition, more than $40 \%$ of doctors report emotional exhaustion or depersonalization, which might impact their willingness to adopt innovations [53]. Therefore, the climate for innovations when considering new healthcare services, require additional funding which might be turbulent [54].

In Greece, the role of gatekeeper connecting patients to the various service providers belongs to pulmonologists. This is a major concern, as they are heavily overburdened by work [22]. In most European countries, the gatekeeper role belongs to primary care providers [22]. Primary care in Greece is mainly responsible for identifying new cases. However, more than $50 \%$ of new COPD patients are not correctly diagnosed, resulting in over-diagnosed cases culminating in unnecessary medication costs [55]. We consider the economic crisis to have a silver lining for the fortuitous timing to advocate for changes that would be beneficial to a variety of healthcare stakeholders [56]. Potential care provision benefits could include allowing integration of specialized nurses into clinical teams or implementing $\mathrm{TH}$ for COPD self-management. While nurses can obtain specialized training in Greece, they are not allowed by law to perform the more specialized tasks they are trained to perform. Considering that TH implementation necessitates the introduction of new roles by nurses, the law limitation for nurses in Greece may endanger $\mathrm{TH}$ sustainability. Our interviews indicate a certain level of readiness for change from the HCPs as they see many problems in the current environment and consider $\mathrm{TH}$ as a tool to help manage them.

Interestingly, in Greece, local healthcare providers are actively participating in $\mathrm{TH}$ efficacy trials for COPD management [57, 58]. Indicating that $\mathrm{HCPs}$ would already have well-formed opinion towards implementing $\mathrm{TH}$. Considering $\mathrm{TH}$ implementation, the right profile of stakeholders is important. Patients should be willing and able (for example, concerns about patient IT literacy) to use technologies, and HCPs should be educated to use $\mathrm{TH}$ and allocated patient data revision as part of care practice.

\section{Strengths and limitations}

This study represents a single case study from a COPD care pathway in Greece, and has certain limitations. First, the use of the CDVC framework has not been applied for COPD disease management in the literature previously. Although several justifications were made for suitability, there is some risk to applying methodological approaches in new contexts. Ideally, testing the CDVC framework for mapping clinical guideline accuracy would have taken place as an individual research method protocol. 
Second, the sample size of four HCPs may be considered small. However, each role in the COPD care pathway of Asclepius was represented, providing rich data material. The gathered information is limited by the lack of ethnographic details in the current study, impacting the external validity of the study [59].

Third, the authenticity of this study was validated independently by the four interviewed HCPs. Even though some English language fluency was a prerequisite for the semi-structured interview, some terminology was difficult to understand for some stakeholders. Due to this, the chief pulmonologist participated in the discussions and helped to facilitate the interviews. This participation may have led to a discrepancy between a given answer in Greek and its translation.

Fourth, some of HCPs have participated in TH pilot studies which may have influenced their perceptions of $\mathrm{TH}$. However, we consider this experience with $\mathrm{TH}$ to be beneficial for our research as they had a wider variety of opinions.

\section{Conclusions}

Although the opportunities and challenges of delivering care to COPD patients are similar across Europe, the solutions to tackle these challenges may require very different approaches. Future research should aim to develop an in-depth understanding of the opportunities for $\mathrm{TH}$ and challenges of $\mathrm{TH}$ implementation in care pathways through longitudinal and complementary data collection methods, such as ethnography. For optimal results, the attitudes and perceptions of HCPs who use these $\mathrm{TH}$ tools should be considered as they provide indepth and practical insights. Moreover, additional stakeholders, such as patients and their relatives, should participate in the research activities to provide a more holistic picture. In the country-specific setting of Greece, tailored $\mathrm{TH}$ solutions should be implemented in care pathways to support HCPs and patients with the management of comorbidities and self-management.

\section{Supplementary information}

Supplementary information accompanies this paper at https://doi.org/10. 1186/s12911-020-01221-y.

Additional file 1. Visualized Representation of CDVC tool for the care practice mapping. Each disease severity category had a different tool, in total four tools, distributed for 4 clinical stakeholders. Each stakeholder should report which procedure they provide to the patient in the table's cross-section.

Additional file 2. Visualized GOLD Guideline Mapping Summary. IC= Integrated Care; TH = Telehealth; *Special Conditional Notes if patient is Exacerbating 1 Resting chronic hypoxemia for Long-term oxygen therapy 2 Non-invasive ventilation 3 Continuous positive airway pressure 4 Chronic Care Model 5 https://www.ncbi.nlm.nih.gov/pubmed/24127811 6 https://www.ncbi.nlm.nih.gov/pubmed/2713226

Additional file 3. Visualized Representation of Policy and Practice. ABCD are the GOLD COPD disease classification categories. White represents exact or similar practices to policy; blue, some absent practices to policy; orange pattern, no similarity from practice to policy; white with crosses, no responses.

Additional file 4. Semi-Structured Interview Guide.

\section{Acknowledgements}

The authors sincerely thank and acknowledge the four HCPs who participated in this study. We thank Philips Research employees for their ideas while designing the interview guide. The perceptions expressed in this publication are those of the HCPs and not necessarily those of the Department of the Hospital.

\section{Endnotes}

${ }^{1}$ For ease of referencing, we will refer to the case study site as 'Asclepius' for anonymization purposes, and to prevent revealing the identity of the hospital and/or staff.

\section{Authors' contributions}

VG, CG, IC and MI developed and designed the study concept. IC facilitated the recruitment of the four HCPs. VG, CG, IC created an interview guide. VG performed the interviews. VG and CG transcribed and analyzed the collected data. HS, SW, MI, IC, NM provided critical insights. All authors approved and contributed to the final written manuscript.

\section{Funding}

Authors VG and CG are funded from the European Union's Horizon 2020 research and innovation programme - Marie Sklodowska-Curie Actions Grant Agreement No. 676201 - CHESS - Connected Health Early Stage Researcher Support System. Funding body had no influence on the design of the study, analysis of the data, the interpretation of the findings or the content of this manuscript.

\section{Availability of data and materials}

The datasets generated during and/or analyzed during the current study are not publicly available to prevent disclosure of personal information.

\section{Ethics approval and consent to participate}

All potential participants were informed in advance about the study in writing and provided with and signed an informed consent form. No ethical declaration was deemed necessary by the internal ethical processes of Philips as no personal data was disclosed. The informed consent forms are managed by the principal researcher (VG). Collection and storage of personal data are in accordance with the General Data Protection Regulation requirements.

\section{Consent for publication}

Not applicable.

\section{Competing interests}

Authors VG and HS are employees of Philips research, Eindhoven, the Netherlands. Author SW is employee of Philips Research, Aachen, Germany.

\section{Author details}

'Laboratory of Computer Science, Medical Informatics and Biomedical Imaging Technologies, School of Medicine, Faculty of Health Sciences, Aristotle University of Thessaloniki, 54124 Thessaloniki, Greece. ${ }^{2}$ Department of Collaborative Care Solutions, Philips Research, High Tech Campus 34, 5656AE Eindhoven, The Netherlands. ${ }^{3}$ M3S, Faculty of Information Technology and Electrical Engineering, University of Oulu, Pentii Kaiteran katu 1, 8000, Fl-90014 Oulu, Finland. ${ }^{4}$ Department of Collaborative Care Solutions, Philips Research, Pauwelsstraße, 1752074 Aachen, Germany. ${ }^{5}$ Department of IEMS,McCormick School of Engineering, Northwestern University, Evanston, IL, USA. 
Received: 24 May 2019 Accepted: 16 August 2020 Published online: 10 September 2020

\section{References}

1. Afonso ASM, Verhamme KMC, Sturkenboom MCJM, Brusselle GGO. COPD in the general population: prevalence, incidence and survival. Respir Med. 2011;105:1872-84

2. Global Strategy for the Diagnosis, Management, and Prevention of COPD. 2018. doi:https://doi.org/10.1097/00008483-200207000-00004.

3. Casas A, Troosters T, Garcia-Aymerich J, Roca J, Hernndez C, Alonso A, et al. Integrated care prevents hospitalisations for exacerbations in COPD patients. Eur Respir J. 2006;28:123-30.

4. John Gibson G, Loddenkemper R, Lundbäck Bo, Sibille Y. Respiratory health and disease in Europe: the new European Lung White Book. Eur Respir J. 2013;42(3):559-63.

5. COPD management. World Health Organization http://www.who.int/ respiratory/copd/management/en/. Accessed 15 Aug 2018.

6. Grumbach K. Chronic illness, comorbidities, and the need for medical generalism. Ann Fam Med. 2003;1:4-7

7. Velardo C, Shah SA, Gibson O, Clifford G, Heneghan C, Rutter H, et al. Digital health system for personalised COPD long-term management. BMC Med Inform Decis Mak. 2017:17:19.

8. Ställberg B, Janson C, Larsson K, Johansson G, Kostikas K, Gruenberger JB, et al. Real-world retrospective cohort study ARCTIC shows burden of comorbidities in Swedish COPD versus non-COPD patients. npj Prim Care Respir Med. 2018;28:1-7.

9. Stroetmann KA, Kubitschke L, Robinson S, Stroetmann V, Cullen K, McDaid D. How can telehealth help in the provision of integrated care? World Health Organization. 2010;:39. http://www.euro.who.int/_data/assets/pdf_ file/0011/120998/E94265.pdf. Accessed 15 Aug 2018.

10. Mclean S, Protti D, Sheikh A. Telehealthcare for long term conditions. BMJ. 2011;342:120.

11. Vitacca M, Montini A, Comini L. How will telemedicine change clinical practice in chronic obstructive pulmonary disease? Ther Adv Respir Dis. 2018;12:153-6.

12. Bourbeau J, Farias R. Making sense of telemedicine in the management of COPD. Eur Respir J. 2018:51:1800851.

13. Wootton R. Twenty years of telemedicine in chronic disease management an evidence synthesis. J Telemed Telecare. 2012;18:211-20.

14. Steventon A, Bardsley M, Billings J, Dixon J, Doll H, Hirani S, et al. Effect of telehealth on use of secondary care and mortality: findings from the whole system demonstrator cluster randomised trial. BMJ. 2012;344.

15. Davy C, Bleasel J, Liu H, Tchan M, Ponniah S, Brown A. Effectiveness of chronic care models: opportunities for improving healthcare practice and health outcomes: a systematic review. BMC Health Serv Res. 2015;15:1-11.

16. McGowan P. The challenge of integrating self-management support into clinical settings. Can J Diabetes. 2013;37:45-50.

17. Valentijn DPP. The 3 differences between value based healthcare and triple aim that you need to know. 2018. https://www.essenburgh.com/en/blog/ the-3-differences-between-value-based-healthcare-and-triple-aim-that-youneed-to-know. Accessed 15 Aug 2018.

18. Amelung VE, Stein V, Goodwin N, Balicer R, Nolte E, Suter E. Evaluating complex interventions. In: Handbook Integrated Care: Springer Nature; 2017. p. 297-313. https://www.researchgate.net/publication/318378091_ Evaluating_complex_interventions.

19. Michael E, Porter EOT. Redefining health care: creating value-based competition on results. In: Redefining Health Care: Creating Value-based Competition on Results; 2006.

20. Lodewijckx C, Decramer M, Sermeus W, Panella M, Deneckere S, Vanhaecht K. Eight-step method to build the clinical content of an evidence-based care pathway: the case for COPD exacerbation. Trials. 2012;13.

21. Utens CMA, Maarse JAM, van Schayck OCP, Maesen BLP, Rutten MPMH, Smeenk FWJM. Care delivery pathways for chronic obstructive pulmonary disease in England and the Netherlands: a comparative study. Int J Integr Care. 2012;12:e40 http://www.ncbi.n/m.nih.gov/pubmed/22977431\%0A; http://www.pubmedcentral.nih.gov/articlerender.fcgi?artid=PMC3440249.

22. Kayyali R, Odeh B, Frerichs I, Davies N, Perantoni E, D'Arcy S, et al. COPD care delivery pathways in five European union countries: mapping and health care professionals' perceptions. Int J COPD. 2016:11:2831-8.

23. Stake R. The art of case study research; 1995.
24. Crowe S, Cresswell K, Robertson A, Huby G, Avery A, Sheikh A. The case study approach. BMC Med Res Methodol. 2011;11:100.

25. Grundstrom C, Gaveikaite V, LB, Maglaveras N, Winter S, Chouvarda I. User Profiles of Healthcare Professionals in a Chronic Obstructive Pulmonary Disease Care Pathway. In: PervasiveHealth'19: 13th EAI International Conference on Pervasive Computing Technologies for Healthcare; 2019.

26. Elfil M, Negida A. Sampling methods in Clinical Research; an Educational Review. Emerg (Tehran). 2017;5(1):PMC5325924. https://www.ncbi.n/m.nih. gov/pmc/articles/PMC5325924/.

27. Boynton PM, Greenhalgh T. Selecting, designing, and developing your questionnaire. BMJ. 2004;328:312-5.

28. Olsen $\mathbf{W}$. Triangulation in social research: qualitative and quantitative methods can really be mixed. In: Developments in Sociology. Causeway Press Ltd; 2004. p. 1-30.

29. Oliver DG, Serovich JM, Mason TL. Constraints and opportunities with interview transcription: towards reflection in qualitative research. Soc Forces. 2005

30. Gale NK, Heath G, Cameron E, Rashid S, Redwood S. Using the framework method for the analysis of qualitative data in multi-disciplinary health research. BMC Med Res Methodol. 2013;13:1-8.

31. Global Strategy for the Diagnosis, Management, and Prevention of COPD. 2017. doi:https://doi.org/10.1164/rccm.201701-0218PP.

32. Sen E, Guclu SZ, Kibar I, Ocal U, Yilmaz V, Celik O, et al. Adherence to GOLD guideline treatment recommendations among pulmonologists in Turkey. Int J Chron Obstruct Pulmon Dis. 2015;10:2657-63. https://pubmed.ncbi.nlm. nih.gov/26715844/.

33. Tsiligianni I, Kampouraki M, lerodiakonou D, Poulonirakis I, Papadokostakis P, Lintovoi E, Karanassos D, Maltezis K, Chorti M, Petrovitsos E, Dimopoulou S, Hamind S, Gialamas I, Athanasiou P, Bempi VLI. COPD patients ' characteristics, usual care, and adherence to guidelines : the Greek UNLOCK study. Int J COPD. 2019;14:547-56.

34. Majothi S, Jolly K, Heneghan NR, Price MJ, Riley RD, Turner AM, et al. Supported self-management for patients with copd who have recently been discharged from hospital: a systematic review and meta-analysis. Int J COPD. 2015;10:853-67.

35. Yang F, Xiong Z-F, Yang C, Li L, Qiao G, Wang Y, et al. Continuity of care to prevent readmissions for patients with chronic obstructive pulmonary disease: a systematic review and Meta-analysis. COPD Chronic Dis. 2017;14:251-61.

36. Janke $R$, Tetrault M, Ferrier M, Burton $L$, Rush $K L$, Hatt $L$. The efficacy of telehealth delivered educational approaches for patients with chronic diseases: a systematic review. Patient Educ Couns. 2018;101:1310-21.

37. Pinnock H, Hanley J, McCloughan L, Todd A, Krishan A, Lewis S, et al. Effectiveness of telemonitoring integrated into existing clinical services on hospital admission for exacerbation of chronic obstructive pulmonary disease: researcher blind, multicentre, randomised controlled trial. BMJ. 2013;347:66070

38. Farmer A, Williams V, Velardo C, Shah SA, Yu LM, Rutter $H$, Jones L, Williams N, Heneghan C, Price J, Hardinge M, Tarassenko L. Self-Management Support Using a Digital Health System Compared With Usual Care for Chronic Obstructive Pulmonary Disease: Randomized Controlled Trial. J Med Internet Res. 2017;19(5):e144.

39. Benjamin Waschki, Anne Kirsten, Olaf Holz, Kai-Christian Müller, Thorsten Meyer, Henrik Watz, Helgo Magnussen, Physical Activity Is the Strongest Predictor of All-Cause Mortality in Patients With COPD. Chest. 2011;140(2): $331-42$

40. Lundell S, Holmner A, Rehn B, Nyberg A, Wadell K. Telehealthcare in COPD: a systematic review and meta-analysis on physical outcomes and dyspnea. Respir Med. 2015;109:11-26.

41. Demeyer H, Louvaris Z, Frei A, Rabinovich RA, de Jong C, Gimeno-Santos E, et al. Physical activity is increased by a 12-week semiautomated telecoaching programme in patients with COPD: a multicentre randomised controlled trial. Thorax. 2017;72:415-23. https://doi.org/10.1136/thoraxjnl2016-209026.

42. Priori R, Van Genugten L, Barretto C, Schonenberg H, Stut W, Miller B, et al. Late breaking abstract - automated coaching for physical activity in COPD patients: results from a pilot study. In: ERS conference; 2017.

43. Harvard School of Public Health THM of H and SS, Education THM of, Affairs $\mathrm{LL}$ and $\mathrm{R}$, and The Hellenic Anti-Cancer Society. The Greek Tobacco Epidemic. 2011. http://www.who.int/fctc/reporting/party reports/greece annex1_the_greek_tobacco_epidemic_2011.pdf. Accessed 19 Aug 2020. 
44. Myung S-K, McDonnell DD, Kazinets G, Seo HG, Moskowitz JM. Effects of web- and computer-based smoking cessation programs. Arch Intern Med. 2009;169:929.

45. Jenny Ure, Hilary Pinnock, Janet Hanley, Gillian Kidd, Emily McCall Smith Alex Tarling, Claudia Pagliari, Aziz Sheikh, William MacNee, Brian McKinstry, Piloting tele-monitoring in COPD: a mixed methods exploration of issues in design and implementation. Prim Care Respir J. 2011;21(1):57-64.

46. Brunton L, Bower P, Sanders C, Soyer HP. The contradictions of Telehealth user experience in chronic obstructive pulmonary disease (COPD): a qualitative Meta- synthesis. PLoS One. 2015;10. https://pubmed.ncbi.nlm.nih. gov/26465333/.

47. Kyriopoulos II, Zavras D, Skroumpelos A, Mylona K, Athanasakis K, Kyriopoulos J. Barriers in access to healthcare services for chronic patients in times of austerity: an empirical approach in Greece. Int J Equity Health. 2014;13:1-7.

48. Huniche L, Dinesen B, Carl Nielsen C, Ove Grann, Egon Toft, Patients' Use of Self-Monitored Readings for Managing Everyday Life with COPD: A Qualitative Study. Telemedicine and e-Health. 2013;19(5):396-402.

49. Chang H. Evaluation framework for telemedicine using the logical framework approach and a fishbone diagram. Healthc Inform Res. 2015;21:230-8.

50. Schonenberg H, Chouvarda I, Filos D, Gaveikaite V, van der Heijden R, Lourida K, et al. Evaluation Engine Architecture Architecture to analyse and monitor scaling-up for integrated care programs. 2016. p. 12-5. https:// www.act-at-scale.eu/wp-content/uploads/2014/08/D4.1-Evaluation-EngineArchitecture.pdf. Accessed 20 Aug 2018.

51. Oderkirk J. Findings of the 2016 OECD HCQI Study of Electronic Health Record System Development and Data Use 2017. http://www.oecd.org/els/ health-systems/health-working-papers.htm. Accessed 20 Aug 2018.

52. Overview of the national laws on electronic health records in the EU Member States: Final report and recommendations. 2014. https://ec.europa. eu/health/ehealth/projects/nationallaws_electronichealthrecords_en. Accessed 10 May 2019

53. Rachiotis G, Kourousis C, Kamilaraki M, Symvoulakis EK, Dounias G, Hadjichristodoulou C. Medical supplies shortages and burnout among greek health care workers during economic crisis: a pilot study. Int J Med Sci. 2014;11:442-7.

54. Kondilis E, Giannakopoulos S, Gavana M, lerodiakonou I, Waitzkin H, Benos A. Economic crisis, restrictive policies, and the population's health and health care: the greek case. Am J Public Health. 2013;103:973-80.

55. Spyratos D, Chloros D, Michalopoulou D, Sichletidis L. Estimating the extent and economic impact of under and overdiagnosis of chronic obstructive pulmonary disease in primary care. Chron Respir Dis. 2016;13:240-6.

56. Filippidis FT, Gerovasili V, Millett C, Tountas Y. Medium-term impact of the economic crisis on mortality, health-related behaviours and access to healthcare in Greece. Sci Rep. 2016;2017(7):1-7.

57. Chouvarda I, Philip NY, Natsiavas P, Kilintzis V, Sobnath D, Kayyali R, Henriques J, Paiva RP, Raptopoulos A, Chételat OMN. WELCOME innovative integrated care platform using wearable sensing and smart cloud computing for COPD patients with comorbidities. In: Annual International Conference of the IEEE Engineering in Medicine and Biology Society; 2014. p. 3180-3.

58. Vasilopoulou M, Papaioannou Al, Chynkiamis N, Vasilogiannakopoulou T, Spetsioti S, Louvaris Z, et al. Effectiveness of home telerehabilitation on functional capacity and daily physical activity in COPD patients. In: European Respiratory Journal; 2015.

59. Yin R. Case study research: design and methods. London: Sage; 2009.

\section{Publisher's Note}

Springer Nature remains neutral with regard to jurisdictional claims in published maps and institutional affiliations.

Ready to submit your research? Choose BMC and benefit from:

- fast, convenient online submission

- thorough peer review by experienced researchers in your field

- rapid publication on acceptance

- support for research data, including large and complex data types

- gold Open Access which fosters wider collaboration and increased citations

- maximum visibility for your research: over $100 \mathrm{M}$ website views per year

At BMC, research is always in progress.

Learn more biomedcentral.com/submissions 